\title{
EXPERIENCIAS DE REGENERACIÓN URBANA Y GESTIÓN DEL RIESGO EN CHILE
}

\author{
Sarmiento Prieto, Juan Pablo \\ (Extreme Events Institute, Florida International University) jsarmien@fiu.edu \\ Fritis Estay, Antonio \\ (Secretaría Ejecutiva Desarrollo de Barrios, Ministerio de Vivienda y Urbanismo) afritis@minvu.cl \\ Castro Correa, Carmen Paz \\ (Departamento de Geografía, Universidad de Chile) y cpcastro@uchilefau.cl
}

\begin{abstract}
RESUMEN
Desde el año 2006 el Ministerio de Vivienda y Urbanismo (MINVU) de Chile avanzó en un proceso de regeneración urbana, entendida como una recuperación de áreas urbanas consolidadas que han experimentado un deterioro social y físico, sectores o barrios de la ciudad que comparten problemas de precariedad, acceso, inequidad, exclusión y frecuentemente exposición a riesgos socionaturales. Por más de 10 años el programa "Quiero mi barrio", ha implementado un proceso participativo tendiente a mejorar la calidad de vida de los habitantes del barrio en una iniciativa que involucra al ministerio, a diferentes agencias sectoriales del nivel nacional, al municipio y a la propia comunidad para la recuperación de los espacios públicos, el equipamiento y el fortalecimiento del tejido social. Este Programa, en un trabajo colaborativo con la Universidad de Chile y Florida International University, ha incorporado en sus proyectos, la metodología de trabajo de gestión del riesgo, que se refleja en intervenciones seguras que contribuyen a la construcción comunitaria de barrios resilientes al riesgo de desastres. A lo largo de su historia, el programa ha realizado numerosas intervenciones de mejoramiento barrial a lo largo del país, cuyos resultados han sido evaluados caso a caso, pero no en sus efectos globales y en el impacto de esta política pública a nivel país.
\end{abstract}

Palabras clave: Programa Quiero Mi Barrio, Intervención urbana, reducción del riesgo de desastres, comunidad.

Bloque temático: Análisis y proyecto territorial.

\begin{abstract}
Since 2006, the Ministry of Housing and Urban Development (MINVU) of Chile has advanced in a process of urban regeneration, understood as a recovery of consolidated urban areas that have experienced a social and physical deterioration, sectors or neighborhoods of the city that share problems of precariousness, access, inequality, exclusion, and frequently exposure to socionatural risks. For more than 10 years the program "Quiero mi barrio" has implemented a participatory process aimed at improving the quality of life of the neighborhood's inhabitants in an initiative that involves the ministry, different sector agencies at the national level, the municipality and the community itself for the recovery of public spaces, infrastructure, and the strengthening of the social fabric. This Program, in a collaborative work with the University of Chile and Florida International University, has mainstreamed risk management methodology in its projects, which is reflected in safe interventions that contribute to the community construction of neighborhoods that are resilient to disaster risk. Throughout its history, the program has carried out numerous neighborhood improvement interventions all over the country, which results have been evaluated on a case-by-case basis, but not in its overall effects nor in the impact of this public policy at the national level.
\end{abstract}

Keywords: Programa Quiero Mi Barrio, Urban intervention, disaster risk reduction, community. Topic: Analysis and territorial project. 


\section{Objetivo}

El objetivo del presente estudio es dimensionar el nivel de avance del programa en procesos de regeneración urbana en Chile, que incorporan propuestas innovadoras en la reducción de riesgo de desastre, así como evaluar su rol en procesos de rehabilitación y reconstrucción post desastres.

\section{Hipótesis}

Las hipótesis que subyacen a esta investigación son:

1. Los programas de regeneración urbana constituyen una ventana de oportunidad para enfrentar problemas de exposición a riesgos socionaturales.

2. Los programas de regeneración urbana constituyen un mecanismo óptimo para adelantar procesos de recuperación pos-eventos socionaturales.

3. El marco legal dispuesto para el programa Quiero mi Barrio permite cumplir adecuadamente su misión.

\section{Antecedentes}

El programa "Quiero Mi Barrio" pertenece al MINVU, nace el año 2006 y basa su trabajo en las interacciones con la comunidad, el municipio y equipos barriales especializados, y busca facilitar el encuentro, la participación y la vida barrial, mediante la recuperación física y social a partir de un diagnóstico integral que fortalece aún más el vínculo de éste con la comunidad. Hasta el año 2016, el Programa ha trabajado con 126 municipios y ha beneficiado a más de un millón de habitantes. Se ha realizado por barrio una inversión cercana a los 497 millones de pesos (US\$753.000), dividiéndose en planes de gestión de obras y social, 395 (US\$598.000) y 102 millones (US\$154.000) respectivamente.

Durante el proceso de implementación de los 520 barrios del Programa en la última década, a lo largo de todo el territorio nacional, han debido hacer frente a una serie de eventos de desastres, lo que llevó a la incorporación de la gestión del riesgo de desastres en su metodología de trabajo. Esto se traduce en diagnósticos territoriales participativos en que se reconocen amenazas, vulnerabilidades y capacidades del territorio, con el fin de construir un Plan Maestro que contribuya a la mitigación del riesgo, definición de vías de evacuación y zonas seguras, así como un Plan de acción comunitario dentro de cada barrio destinado a mejorar la calidad de vida y fortalecer la resiliencia. En este contexto, en relación a los componentes del riesgo que se señalan, se entiende por amenaza la probabilidad de que un evento físico natural o antrópico provoque daño o pérdidas a la población y sus medios de vida; la vulnerabilidad corresponde a la exposición y susceptibilidad de la población a ser afectada por dicho evento, mientras el riesgo es la probabilidad de exceder un nivel de pérdidas o daños, resultante de la condición de amenaza y vulnerabilidad en un territorio determinado.

En el período comprendido entre los años 2006 y 2016, diversos eventos geológicos e hidrometeorológicos afectaron varias zonas del país, y como parte de la reconstrucción de estos territorios, el PQMB se hace presente con el fin de acompañar a la población en este proceso y también, como una forma de contribución al mejoramiento de áreas deteriorados de la ciudad, como una medida de rehabilitación urbana y reducción del riesgo de desastre.

La tabla 1. recoge algunas experiencias significativas del PQMB. En algunos casos el programa estaba presente antes del evento, en otros fue llamado a intervenir. 
Tabla 1. Experiencias significativas del Programa Quiero mi Barrio

\begin{tabular}{|l|l|l|}
\hline Anos & Evento & Acción PQMB \\
\hline 2007 & Terremoto que afectó el Norte Grande del país 7.7 Mw. & Reubicación comuna de Tocopilla \\
\hline 2010 & $\begin{array}{l}\text { Terremoto 8.8 Mw que afectó la zona centro sur del país. } \\
802 \text { fallecidos, cerca de 820.000 damnificados y cerca de } \\
400.000 \text { viviendas con daños de diversa consideración. }\end{array}$ & $\begin{array}{l}\text { El PQMB incorpora } \\
\text { decisididamente el proceso de } \\
\text { reconstrucción }\end{array}$ \\
\hline 2014 & $\begin{array}{l}\text { Terremoto que afectó al país pero en el extremo norte, } \\
\text { con una magnitud de 8.2 Mw. }\end{array}$ & $\begin{array}{l}7 \text { nuevos barrios de interés regional } \\
\text { al PQMB, ubicados en las regiones } \\
\text { de Arica y Parinacota y Tarapacá. }\end{array}$ \\
\hline 2014 & $\begin{array}{l}\text { Incendios forestales con el mayor impacto de las últimas } \\
\text { décadas en el área urbana de Valparaíso. Afectó 965 } \\
\text { hectáreas, de las cuales 28,5 correspondieron a zonas } \\
\text { habitadas. 2.900 viviendas resultaron destruidas con } \\
12.000 \text { damnificados, 15 fallecidos y 500 heridos. }\end{array}$ & $\begin{array}{l}\text { El PQMB incorpora 4 nuevos barrios } \\
\text { localizados en los cerros de } \\
\text { Valparaíso } \\
\text { completamente quemados }\end{array}$ \\
\hline 2015 & $\begin{array}{l}\text { Aluviones en la región de Atacama. 26 fallecidos, 30.000 } \\
\text { damnificados, 97.000 afectados y cerca de 20.000 } \\
\text { viviendas con daños de diversa magnitud. }\end{array}$ & $\begin{array}{l}\text { El PQMB incorpora 2 nuevos en la } \\
\text { región de Atacama. }\end{array}$ \\
\hline 2015 & $\begin{array}{l}\text { Erupción del volcán Calbuco, en la región de Los Lagos, } \\
\text { y aluvión que afectó la zona norte del país, } \\
\text { específicamente la zona de Tocopilla }\end{array}$ & $\begin{array}{l}\text { El PQMB tenía barrios en ejecución } \\
\text { previo a los eventos. }\end{array}$ \\
\hline 2015 & $\begin{array}{l}\text { Terremoto 8.4 Mw afectó la zona centro norte del país, } \\
\text { con epicentro en Canela Baja. 15 fallecidos, 18.000 } \\
\text { damnificados y 9.800 viviendas resultaron con viviendas } \\
\text { de diversa consideración. }\end{array}$ & $\begin{array}{l}\text { Tres barrios del Programa fueron } \\
\text { afectados por este evento. }\end{array}$ \\
\hline
\end{tabular}

\section{Marco Teórico}

Para algunos autores los orígenes del movimiento llamado regeneración urbana se ubican en el Reino Unido hacia inicios de la de década de 1990, extendiéndose rápidamente a otros países europeos (Roberts et al. 2000; Smith, 2011). Según Roberts (2000) la regeneración urbana es una visión y una acción integral que conduce a la resolución de problemas urbanos y que busca lograr una mejora duradera en las condiciones físicas, sociales, económicas y ambientales de un área o territorio que ha estado sujeta a cambios. Para Roberts, la regeneración urbana corresponde a un propósito estratégico a largo plazo, que exige de una integración intersectorial.

Es importante indicar que la regeneración urbana surge en Europa como resultado de un proceso de más de 40 años que inicia con la reconstrucción posterior a la segunda guerra mundial en la década de 1950, que fue seguida de la política de revitalización de los 60 como reacción a la caída de las economías locales, la cual a su vez antecedió a la política de renovación de barrios de los 70, y las tendencias desarrollistas de los 80 . La visión de regeneración de los 90 se nutrió en alguna forma de estas políticas que la precedieron, buscando asociar integralmente política y práctica en intervenciones urbanas (Beswick \& Tsenkova, 2002). Esta asociación a periodos anteriores fue abordada inicialmente por Coach (1990), quién indicó que la regeneración urbana se diferencia de la revitalización (o rehabilitación) ya que ésta última se limita a un método de enfoque único y rígido; trasciende a la renovación urbana, a la que se considera como un proceso de cambio 
esencialmente físico; y va más allá del desarrollo urbano (o reurbanización), pues ésta tiene una misión y un propósito demasiado generales.

Como parte del telón de fondo a todo este proceso de transición de políticas urbanas es necesario hablar del neoliberalismo como modelo económico prevalente a nivel global y el cual tiene su expresión en lo urbano. Mayer (2007) hace referencia a las "ciudades neoliberales" que se conciben bajo políticas favorables al crecimiento económico y al desmantelamiento del estado del bienestar, la justicia social y ambiental, en favor de incentivos a la empresarialidad, la privatización de servicios, la desregulación del uso de la tierra, y la construcción de megaproyectos atractivos para la inversión. Los resultados saltan a la vista: mayor autonomía municipal con exiguos presupuestos, crecimiento de los mercados formales e informales de la tierra y la vivienda, deterioro de los barrios de vivienda popular y disminución de las inversiones sociales. Paralelamente aparecen discursos sobre el deterioro urbano y la necesidad de abordarlo a través de una renovación, con el consecuente efecto: gentrificación. Como dice Mayer, "la neoliberalización de la ciudad ha creado de muchas maneras un entorno más hostil y más difícil para el progreso de los movimientos urbanos" (Mayer, 2012, p. $69)$.

En Inglaterra la regeneración urbana ha sido y continúa en los últimos cuarenta años. Esta estrategia se ha abordado a través de políticas económicas y de planificación orientadas a la renovación física y económica, reconociendo la imperiosa necesidad de incorporar políticas sociales y ambientales, esenciales para conseguir una revitalización urbana con un enfoque integral (Coach, 1999).

Como toda teoría, hay algunos principios de la regeneración urbana que marcan una clara diferencia con otros enfoques:

- Basarse en un análisis de las condiciones locales.

- Establecer objetivos claros y cuantificados.

- Enfocar un cambio integral en el tejido físico, estructuras sociales, base económica y condiciones ambientales.

- Ser coherente con los objetivos del desarrollo sostenible.

- Hacer el mejor uso posible de los recursos naturales, económicos, humanos y otros disponibles.

- Buscar la participación y el consenso entre las partes interesadas.

- Medir el progreso de la estrategia y supervisar las fuerzas internas y externas cambiantes que actúan en las áreas locales.

- Reconocer que diferentes elementos de la estrategia progresarán a diferentes velocidades.

Indudablemente hay en los temas de regeneración urbana un componente que busca el abordaje de una degradación física asociada a aspectos de deterioro social, económico y ambiental, circunstancias que han hecho que ciudades alrededor del mundo, particularmente en países desarrollados, hayan tenido que reinventarse. Un tema de interés compartido por ciudades europeas, norteamericanas y de algunos países que ingresan más recientemente al grupo del mundo industrializado, es el de la inmigración. Por un lado, muchos gobiernos locales buscan un tipo de inmigración representado por grupos sociales altamente calificados, con capacidad empresarial y creativa. Sin embargo, hay una discrepancia en los flujos migratorios que se experimentan en las últimas dos décadas de este siglo, donde se observa una afluencia no planificada, poco calificada, sin capacidad de inversión y frecuentemente etiquetada dentro de la ilegalidad.

Para Latinoamérica, hay un factor de gran importancia que no es mencionado en la literatura revisada sobre el tema de regeneración urbana: las amenazas naturales y antrópicas. No se encontraron referencias a los aspectos potenciales que derivan de una exposición a estas amenazas, ni tampoco al impacto que éstas generan cuando se materializan. Este estudio busca explorar la experiencia del programa de regeneración urbana del MINVU, conocido como Quiero mi Barrio, en su forma de abordar ambas circunstancias.

Para la construcción del marco teórico de este estudio se ha empleado la propuesta de sistema de Tsenkova (2001), con algunos cambios que permiten comprender la problemática urbana sobre la cual actúa la propuesta de regeneración. La figura 1 ilustra posibles factores de cambio negativos involucrados en los cambios 
experimentados a nivel de un territorio en particular, pudiendo ser estos el resultado de tendencias económicas (neoliberalismo), sociales (movilidad social), y espaciales (abandono de centros urbanos, preferencia por periferias); o consecuencia de cambios a nivel de la gobernanza local (interacción gobierno local, sociedad civil y comunidad), tecnología (mercados y banca online), y políticas nacionales (regulación urbano-rural, incentivos al sector de la construcción). Otros aspectos que pueden influir en el territorio son las amenazas, las cuales tienen un carácter potencial (amenazas o antrópicas); o bien estas amenazas podrían materializarse en situaciones de emergencia o desastre con el respectivo impacto (impacto directo e indirecto); y los efectos secundarios evidentes en los grandes agregados macroeconómicos como inflación, crecimiento económico, desequilibrios en la balanza de pagos, incremento del gasto público.

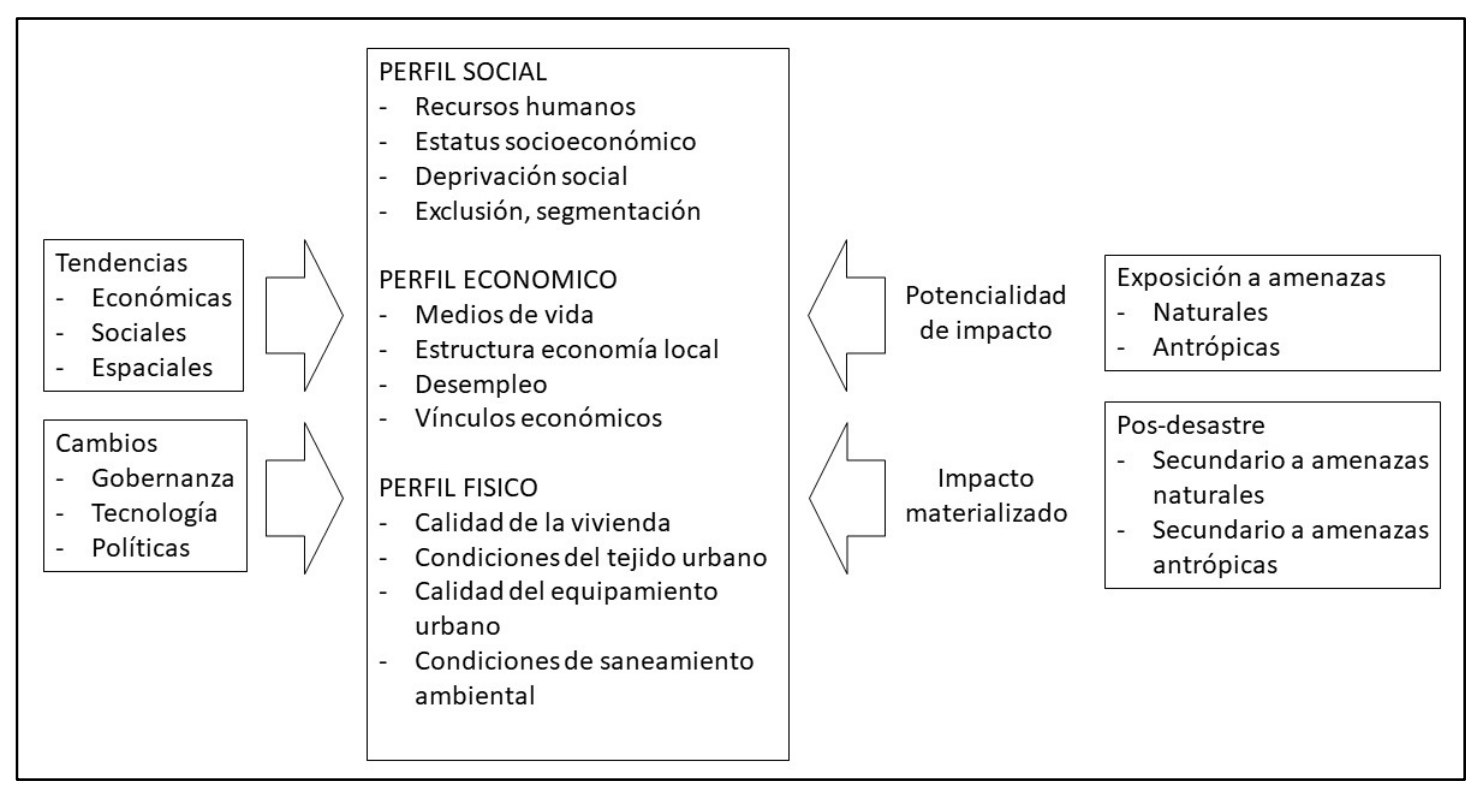

Fig. 1 Modelo Factores de Cambio involucrados en la Precariedad e Informalidad, Fuente: Elaboración a partir del modelo propuesto por Tsenkova, 2001.

En el centro de la figura 1 se encuentra la comunidad analizada ubicada en una unidad territorial específica, la cual se caracteriza a través de las tres esferas: social (incluye el análisis de recursos humanos, estatus socioeconómico, deprivación social, exclusión, segmentación); económica (contempla los medios de vida, estructura de la economía local, desempleo, vínculos económicos; y la física (abarca la calidad de la vivienda, las condiciones del tejido urbano, calidad del equipamiento urbano, condiciones de saneamiento ambiental).

El modelo de factores de cambio expuesto ofrece el escenario en el cual interactúa la propuesta de regeneración urbana, particularmente el proyecto de regeneración del PQMB del MINVU de Chile.

\section{Metodología}

El estudio utilizó una metodología de investigación basada en evidencia (IBE), construida sobre un análisis sistemático del desempeño del PQMB, y las intervenciones reales llevadas a cabo durante la vida del programa. Se analizaron un total de 520 proyectos distribuidos en 16 regiones, de los que se seleccionaron casos representativos para los objetivos propuestos. Se caracterizaron los barrios en función de su exposición a amenazas, existencia de planes reguladores en las poblaciones, encontrarse en la fase de implementación del proyecto, indicadores socioeconómicos y otros. El diseño de la investigación incluye una revisión de literatura, seguida de métodos de investigación mixtos, que consideran enfoques cualitativos y cuantitativos: georreferenciación y análisis de patrones urbanos, una revisión exhaustiva de los registros sobre intervenciones físicas, ambientales y sociales relevantes llevadas a cabo en los proyectos seleccionados. 


\subsection{Selección de casos de estudio}

En el contexto de la relación entre las intervenciones del Programa Quiero mi Barrio (PQMB) y la reducción del riesgo de desastres, debido a la gran cantidad de barrios del programa a nivel nacional (520), la diversidad de amenazas presentes en el territorio y los múltiples contextos urbanos y geográficos en los que se encuentran emplazados, se optó por la aplicación de dos filtros que permitiesen acotar los casos de estudio a aquellos barrios que se han visto afectados por algún desastre desde la implementación del Programa en el año 2006.

El primer filtro aplicado corresponde a seleccionar aquellos barrios afectados por eventos ocurridos entre el año 2006 y 2016 ya que es el primer periodo de vigencia del Programa y en el cual hubo desastres importantes que marcaron un antes y un después en la gestión del riesgo que se está llevando a cabo en el país. Algunos de estos eventos son terremotos, tsunami, incendios forestales, aluviones y erupciones volcánicas. Luego de este filtro, se obtuvo un total de 33 barrios a nivel nacional.

A continuación, con los barrios resultantes del primer filtro, se aplicó el segundo filtro correspondiente a aquellos barrios que se encuentran en zonas con amenaza de tsunami y/o incendio forestal. La selección de estas amenazas responde a la disponibilidad de información como cartografías y estadísticas relacionadas a los eventos ocurridos entre el año 2006 y 2015, y a la gravedad de sus consecuencias. Una vez aplicado este filtro, son finalmente 11 los barrios seleccionados para la presente investigación.

\subsection{Caracterización de la amenaza de los barrios}

Se trabajó en base a archivo KMZ (Google Earth) enviado por el Programa de Recuperación de Barrios del Ministerio de Vivienda y Urbanismo (MINVU) en el cual aparece la localización espacial de los 520 barrios intervenidos por el PQMB a lo largo de todo el país, el cual fue transformado a archivo vectorial (Shape ArcGis). Al mismo tiempo, se generó base de datos en el software Excel con la información disponible en el archivo KMZ original.

Para la amenaza de inundación por tsunami, se descargaron desde la web de "Infraestructura de Datos

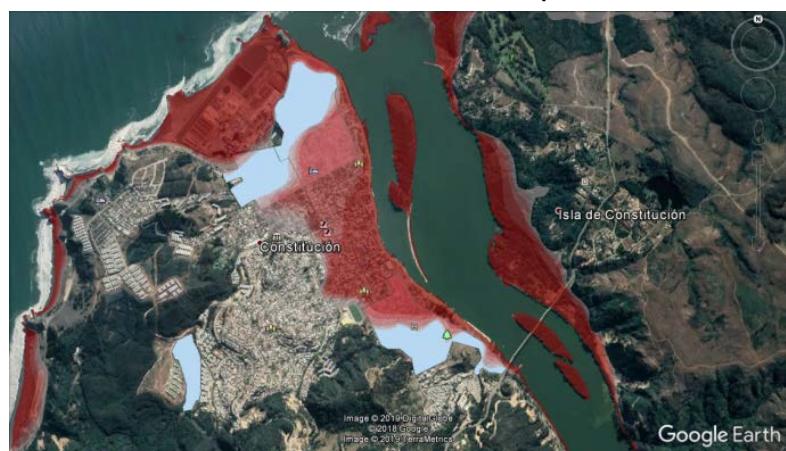

Fig. 02 Carta de inundación. Fuente: (SHOA, 2019)
Espaciales (IDE)" las cartas de inundación por tsunami del país que pertenecen al Servicio Hidrográfico y Oceanográfico de la Armada de Chile (SHOA) en formato KML. En Google Earth se compararon las cartas de inundación con el archivo KMZ original de los barrios y se agregó a la base de datos correspondiente (Ej. Fig 2), dependiendo si el barrio se encontraba en zona de inundación o bien, en zona de evacuación (cota 30 m.s.n.m.).

Para la amenaza de incendio forestal, se comparó la localización de cada barrio con la amenaza de incendio forestal mediante el software ArcGis y se agregó a la base de datos si el barrio se encuentra en una zona de "Alta" o "Baja" amenaza de incendio. 

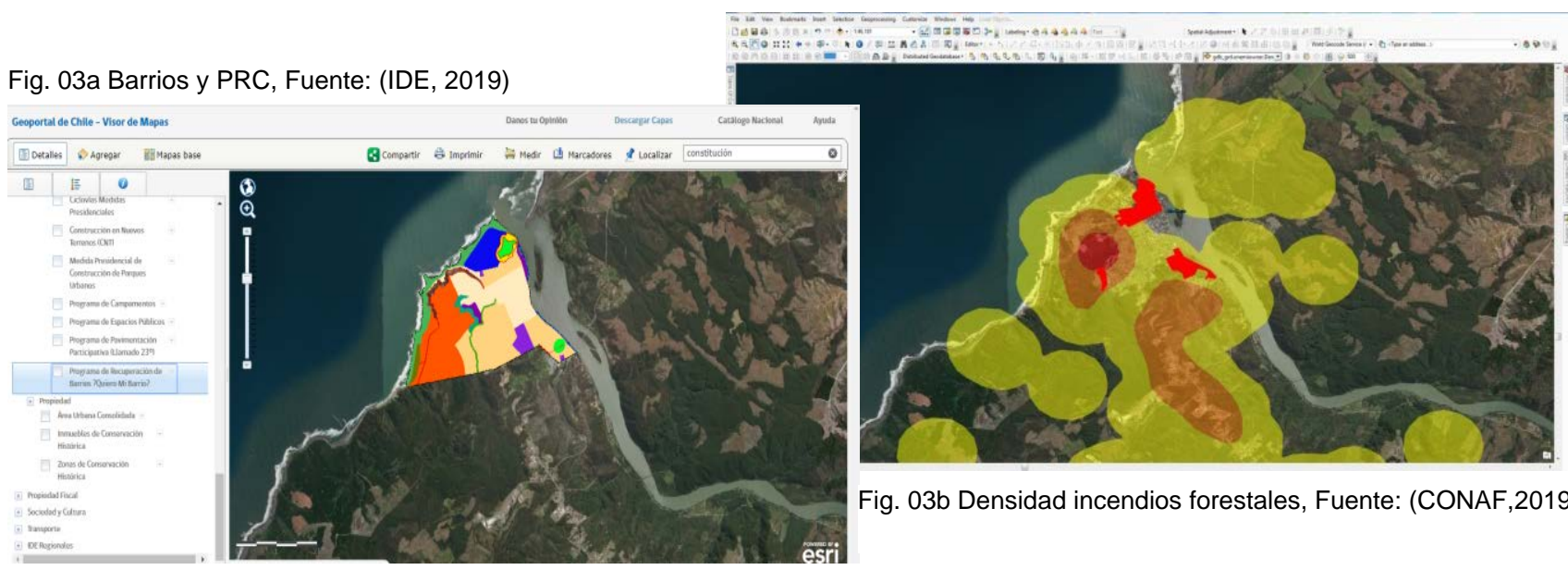

Fig. 03b Densidad incendios forestales, Fuente: (CONAF,2019)

Finalmente, con base en la página web de Infraestructura de Datos Geoespaciales de Chile (IDE-Chile) se comparó la localización de los barrios con el Plan Regulador Comunal (PRC) correspondiente y se completó la base de datos referida a si el barrio se encuentra emplazado en una zona en la que existe PRC y "Usos" en el que se agregó todos aquellos usos indicados en el instrumento de planificación que se encuentran dentro de los límites del barrio (Fig 3a y 3b).

\section{Resultados y Discusiones}

Este artículo sistematiza la experiencia del MINVU en este campo, ofreciendo una base de evidencias que pueden ser empleadas en la formulación de políticas de desarrollo de otros países de la región de las Américas. EI PQMB ha impactado como política pública la forma de gestión territorial, articulando bajo un modelo de intervención al gobierno regional, gobierno local y ciudadanía, mediante un modelo de urbanismo ciudadano que ha sido exitoso por la coproducción participativa a lo largo de todo el proceso de diagnóstico, diseño y ejecución de proyectos físico sociales, estableciendo los derechos y deberes de cada una de las partes impactando al modelo de gobernanza territorial en su fase de prevención y respuesta con el objetivo de que tras un evento la recuperación sea rápida y eficiente.

En los 520 barrios seleccionados a lo largo del país, se han realizado intervenciones que han permitido mejorar la habitabilidad de áreas urbanas deterioradas, reforzando a su vez, el tejido social, la gobernanza y, de forma directa e indirecta, se han generado acciones para reducir el riesgo de desastres y fortalecer la resiliencia de las comunidades, debido a que en muchos casos, los barrios están localizados en entornos de alta exposición a amenazas (Cutter et al., 2008; Morrow, 2008). El análisis de las intervenciones realizadas por el PQMB, consideró sus tres ejes tradicionales de mejoramiento barrial: Eje de identidad y patrimonio, Eje Medio ambiente y Eje de seguridad, bajo el cual se incluyó, la temática de reducción de riesgo de desastres.

Para la selección de los barrios a analizar en este estudió se aplicaron dos filtros, teniendo como punto de partida los 520 barrios trabajados entre el 2006 y el 2016 a lo largo del país. El primer filtro seleccionó los barrios afectados severamente por eventos naturales, resultando 33 barrios. El segundo filtro identificó los barrios impactados por tsunamis e incendios, obteniendo 11 barrios que se convirtieron en la muestra del estudio. Para este segundo filtro se seleccionaron estas dos amenazas debido a disponibilidad de datos a nivel nacional. Se analizó el tipo de proyectos llevados a cabo en los 11 barrios seleccionados en función de aspectos que favorecen la reducción del riesgo de desastres y la resiliencia (Figura 4). 


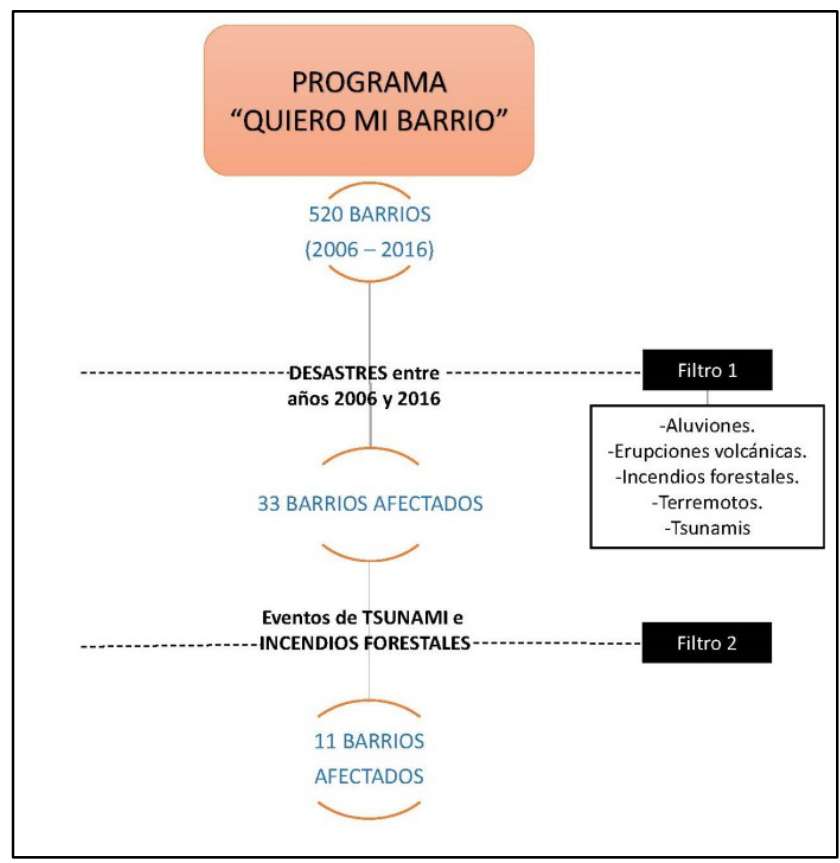

Fig. 04 Flujo selección casos estudio, Fuente: Elaboración propia

A continuación se indican los resultados del análisis de las obras de infraestructura y sociales que se realizaron en los barrios seleccionados del PQMB, además de especificar si el proyecto incluye o no acciones de gestión para la reducción del riesgo de desastres (RRD).

En los proyectos que no contemplaban RRD es evidente que el programa está centrado en el espacio público (Jackson \& Sinclair, 2012) dando énfasis a obras físicas que generan o mejoran áreas de encuentro de la comunidad, a bienes de uso comunitario, de protección del patrimonio histórico y cultural, el incremento de la accesibilidad y las vías de circulación peatonal, de la construcción de espacios de reunión para los vecinos, de áreas verdes, de juegos y esparcimiento, el mejoramiento de la iluminación y el saneamiento sanitario. Estas mejoras tienen un impacto directo e indirecto en la gestión de riesgos. El directo, al asegurar vías amplias que facilitan el acceso de organismos de respuesta y la evacuación de la comunidad en caso de emergencia, o al proporcionar espacios abiertos donde pueda ubicarse la comunidad una vez evacuada.

En lo social, hay un énfasis en los proyectos que fomentan la cohesión social y la generación de redes (FosterFishman et al.,, 2001), los procesos de planificación participativa, fortalecimiento de capacidades en las áreas medioambiental, organizacional, seguridad, así como de desarrollo y formación de oficios, así como el resguardo de la memoria histórica y el enriquecimiento cultural, el cuidado del patrimonio y la gestión de residuos.

Como resultado, más allá del incuestionable mejoramiento físico de los barrios intervenidos, es evidente el impacto social en términos de cohesión, arraigo y apego al territorio, circunstancias que contribuyen significativa e indudablemente a fortalecer el capital social y por ende la resiliencia de estas comunidades.

En siete de los proyectos se va aún más allá, se aborda directamente el tema de gestión de riesgos, tres de ellos expuestos a la amenaza de tsunamis (Regiones de Los Lagos y de Arica y Parinacota) y cuatro a la amenaza de incendios forestales (Región de Valparaíso). En estos casos las obras físicas estuvieron asociadas a la construcción de zonas seguras y de rutas de evacuación, el desarrollo de planes comunitarios de gestión de riesgos y manejo de emergencias, instalaciones estratégicas de seguridad, mejoramientos de la accesibilidad, incorporación de elementos de seguridad en plazas y áreas comunes como iluminación, señalética, redes secas para el combate de incendios, todas acciones que demuestran la factibilidad de implementar proyectos de regeneración urbana integrando la gestión de riesgos, contribuyendo así a la 
seguridad, la resiliencia y la sostenibilidad de los barrios intervenidos, como al fortalecimiento del capital social (Mayunga, 2007; Patel \& Gleason, 2018; Townshend et al., 2015).

Los gráficos 5 y 6 muestran la integralidad de proyectos en lo que respecta a las obras físicas, tanto aquellos que incluyen la gestión de riesgos como aquellos previos que no tienen este componente. Sin excepción todos aportan al mejoramiento del entorno urbano y al bienestar de la comunidad. Sin embargo, es claro que los proyectos que incorporan gestión de riesgos impactan positivamente en un mayor número de aspectos y permiten generar una más amplia diversidad de proyectos que aportan no solo a mejorar la calidad de vida del barrio sino también a su seguridad.

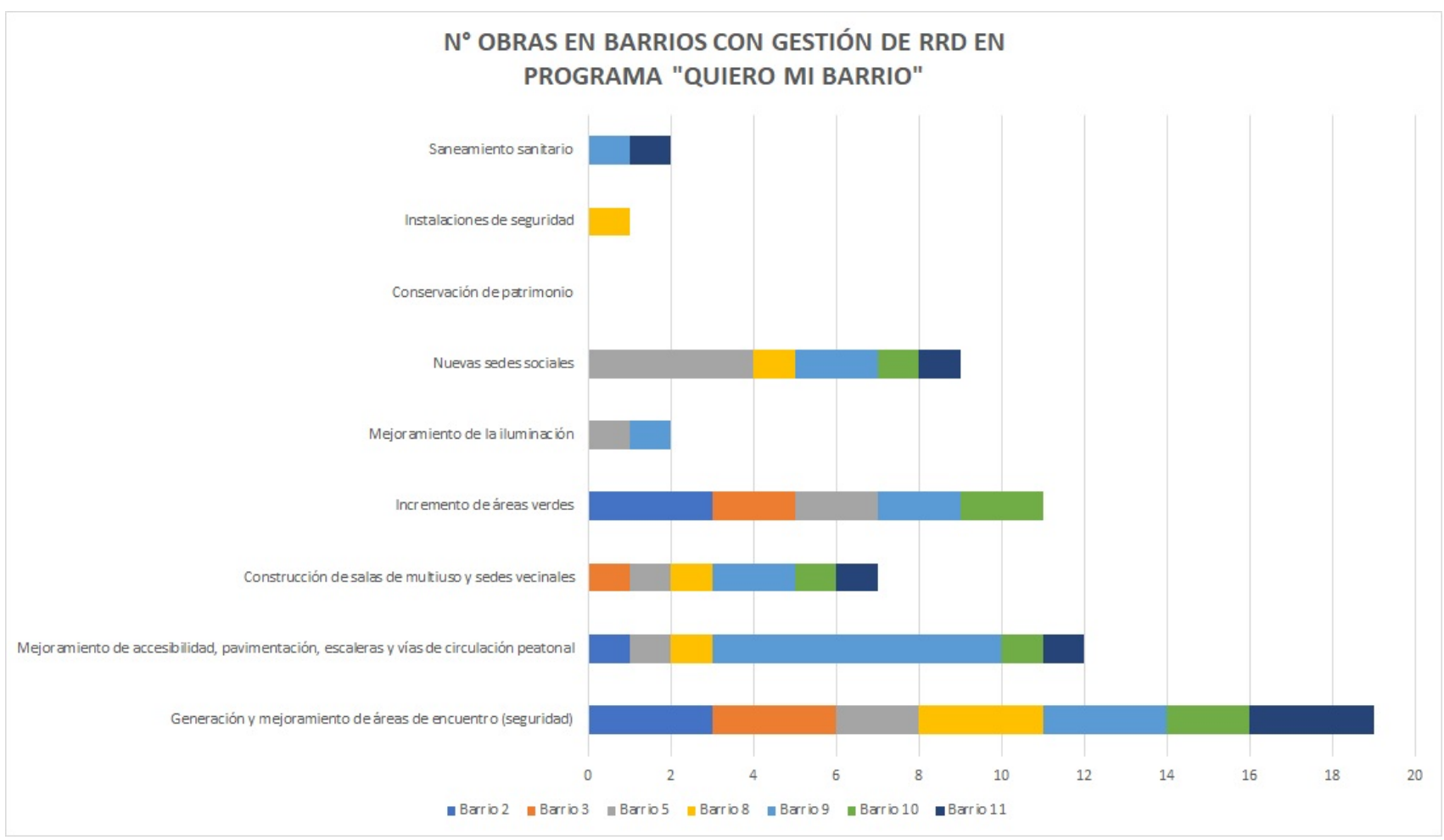

Fig. 05 Obras en barrios donde se implementaron acciones directas de reducción de riesgos Elaboración propia 


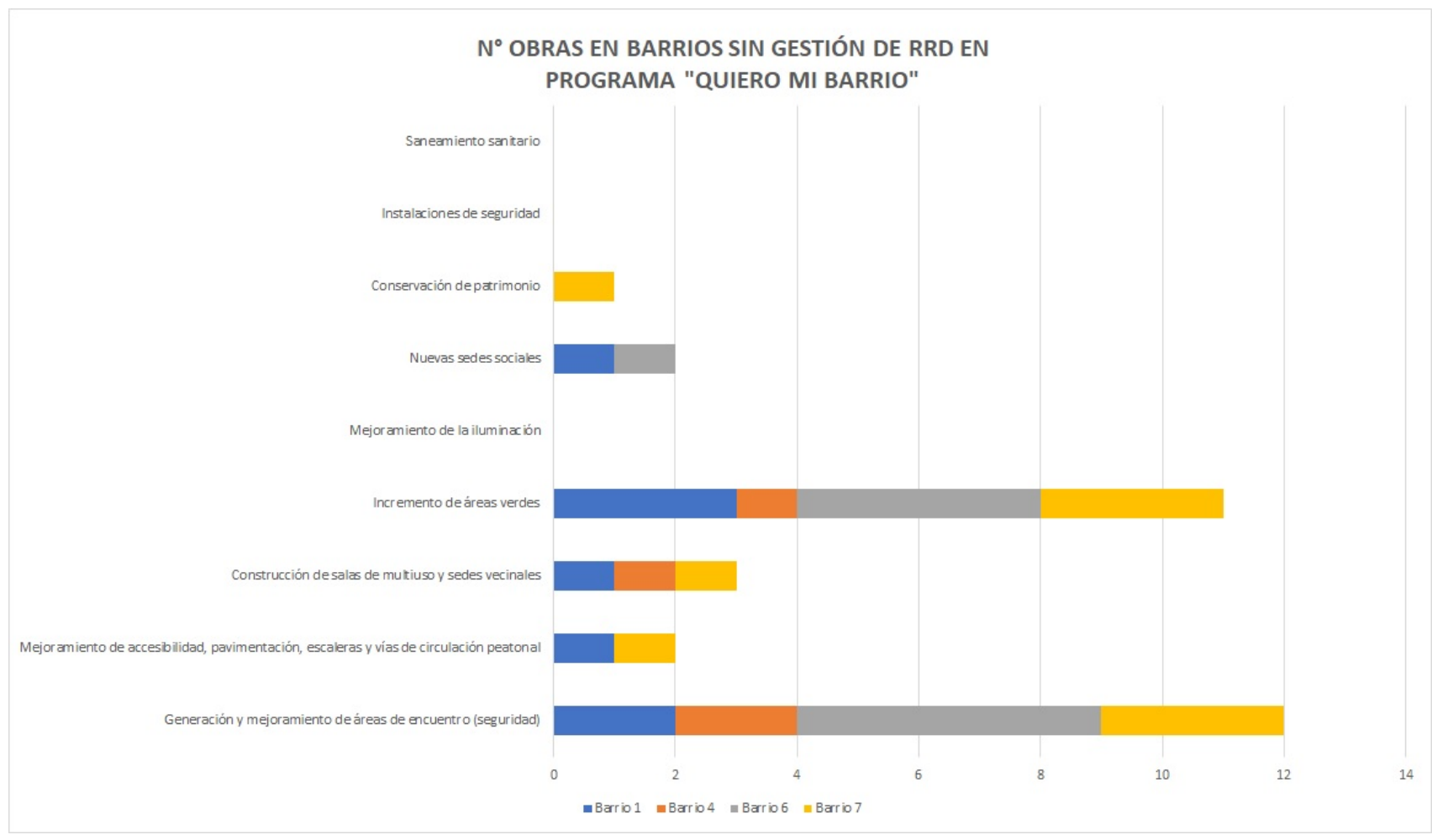

Fig. 06 Obras en barrios donde no se implementaron acciones directas de reducción de riesgos Elaboración propia

\section{Inserción de la gestión del riesgo de desastres en una política pública}

El programa de regeneración urbana PQMB se encuentra regulado directamente por el Decreto Supremo 14 de 2007, y tiene como objetivo mejorar la calidad de vida de los habitantes de barrios que presentan problemas de deterioro urbano, segregación y vulnerabilidad social (MINVU, 2008). A su vez el programa se ubica dentro de la Política Nacional de Desarrollo Urbano del 2013 que fija el escenario mas amplio de acción. A nivel operacional el PQMB cuenta con un Manual de Procedimientos expuesto en la Resolución Exenta N4119 de 2011. Si bien desde sus inicios el programa ha respondido a la demanda de servicios en respuesta a desastres, no fue hasta el año 2016 que se planteó una propuesta de ajuste al Decreto Supremo para integrar la temática del riesgo de desastre, como una tercera causal de intervención del programa. A la fecha esta propuesta de modificación no ha logrado pasar las instancias necesarias para aprobarse, sin embargo, se ha llevado a cabo de una forma funcional y sistemática aprovechando ventanas de oportunidad enmarcadas en el Plan Estratégico Nacional para la Gestión del Riesgo de Desastres 2015-2018 (ONEMI, 2016).

Esta integración se hace manteniendo los techos presupuestales dispuestos para los proyectos que entran a concurso, postulados por las alcaldías. Esto es que sin incrementar el presupuesto se integra transversalmente el concepto de riesgos. Esta estrategia se está implementando desde entonces con los resultados expuestos en este informe. Otros proyectos que tienen un interés regional se ven beneficiados por recursos adicionales de diferentes fuentes de financiación, lo cual permite una mayor inversión en el tema de riesgos. 
1. Barrio Alborada, comuna de Arica, Región de Arica y Parinacota. $\$ 642.5$ millones (US\$973.446)

2. Barrio Jorge Inostroza, comuna de Arica, Región de Arica y Parinacota. $\$ 657.3$ millones (US\$995.869)

3. Barrio Puerta Norte, comuna de Arica, Región de Arica y Parinacota. $\$ 637.3$ millones (US\$965.567)

4. Barrio Gabriela Mistral, comuna de Taltal, Región de Antofagasta. \$952.7 millones (US\$1.443.427)

5. Barrio 21 de Mayo, comuna de Ovalle, Región de Coquimbo. $\$ 647.3$ millones (US\$980.718)

6. Barrio Las cañas, comuna de Valparaíso, Región de Valparaíso. \$1.271.4 millones-Reconstruccion (US\$1.926.286)

7. Barrio Cerro El Litre, comuna de Valparaíso, Región de Valparaíso. \$2.065.3 millones-Reconstrucción (US\$3.129.116)

8. Barrio Cerro La Cruz, comuna de Valparaíso, Región de Valparaíso. \$1.921.0 millones-Reconstrucción (US\$2.910.489)

9. Barrio Merced-La virgen, comuna de Valparaíso, Región de Valparaíso. \$1.969.4 millones-Reconstrucción (US\$2.983.819)

10. Barrio Bosque Nativo Alerce Norte, comuna de Puerto Montt, región de Los Lagos. $\$ 647.3$ millones (US\$980.718)

11. Barrio Centro histórico, comuna de Maullín, Región de Los Lagos. \$941.5 millones (US\$1.426.458)

Fig. 07. Inversiones en los 11 barrios seleccionados

En las situaciones pos-desastre surgen otras figuras jurídicas que apropian recursos adicionales al programa bajo el amparo de la rehabilitación y reconstrucción. De hecho, 4 de los 7 proyectos aquí estudiados (Valparaíso, ver fig 07) se enmarcan en este tipo de financiación y demuestran como con esta inversión adicional se logran niveles apropiados de protección de la comunidad y fortalecimiento de su resiliencia.

\section{Conclusiones}

La estrategia inicial del PQMB del MINVU se enfocó en los aspectos de infraestructura y de carácter social, los cuales apuntaban preferentemente a la construcción y mejoramiento de espacios comunes como sedes vecinales y áreas verdes, entre otros, así como obras sociales que permitían mejorar la organización y cohesión social, además de incentivar la permanencia de la memoria histórica.

Al incorporar el concepto de riesgo en los diseños -tanto en las líneas de obras de infraestructura como en las obras sociales- de mejoramiento urbano del PQMB se lograron proyectos que contribuyen significativamente a la reducción del riesgo ante desastres. Esto impacta directamente en el mejoramiento de la seguridad y la resiliencia del barrio y su comunidad.

A través de este estudio queda claro que aquellos proyectos iniciales del programa que no incorporaban el objetivo de reducir el riesgo de desastres, contribuyen también a incrementar la participación ciudadana, el conocimiento del territorio, el incremento de las áreas verdes y espacios comunes, el mejoramiento de la conectividad, y otras acciones que aportan a la resiliencia de la comunidad ante desastres.

El hecho de incorporar el enfoque de gestión del riesgo en la metodología del programa ha permitido lograr un impacto significativo ya que además del mejoramiento en calidad de vida en el barrio y fortalecimiento del tejido social, se puede reducir el riesgo de desastres con acciones de preparación para la respuesta a emergencias y de prevención de eventos adversos. La incorporación de la reducción de riesgos en los proyectos implica un incremento en el costo que variara segun los factores de exposición, susceptibilidad y tipo de amenaza, pero tendrá claros beneficios para la seguridad de la población, la reducción de perdidas ante futuros eventos y la sostenibilidad de los procesos de desarrollo de estas comunidades.

Es importante avanzar en el estudio de impacto socioeconómico del PQMB para respaldar el proceso de adecuación de la norma que lo rige, a fin de poder actuar ante situaciones de riesgo manifiesto o ante desastres ocurridos con la misma celeridad y efectividad con que actúa ante problemas de deterioro urbano, segregación y vulnerabilidad social. 


\section{BIBLIOGRAFÍA}

BEWICK, C.-A., \& TSENKOVA, S. (2002). Overview of Urban Regeneration Polices. In S. Tsenkova (Ed.), Urban regeneration: learning from the British experience. Calgary, Alberta: Faculty of Environmental Design, University of Calgary.

COUCH, C. (1990). Urban renewal: theory and practice. London: Macmillan Education. Couch, C. (1999). Housing development in the city centre. Planning Practice and Research, (14), 69-86.

CUTTER, S. L., BARNES, L., BERRY, M., BURTON, C., EVANS, E., TATE, E., \& WEBB, J. (2008). A placebased model for understanding community resilience to natural disasters. Global Environmental Change, 18(4), 598-606. https://doi.org/10.1016/i.gloenvcha.2008.07.013 (Consulta: 5/03/2019).

DIAZ, GABRIELA, (2017) Propuesta metodológica para la incorporación de la Reducción del Riesgo de Desastres en la metodología de barrio del Programa "Quiero mi Barrio". Secretaría Ejecutiva de Recuperación de Barrios, MINVU. Informe Final de Práctica Profesional. Universidad de Chile, Santiago.

FOSTER-FISHMAN, P., BERKOWITZ, S., LOUNSBURY, D., JACOBSON, S., \& LLEN, N. (2001). Building collaborative capacity in community coalitions: A review and integrative framework. American Journal of Community Psychology, 29(2), 241-61.

JACKSON, R., \& SINCLAIR, S. (2012). Designing healthy communities (1st ed). San Francisco: Jossey-Bass.

MAYER, M. (2007). Contesting the Neoliberalization of Urban Governance (pp. 90-115). Presented at the Contested urban futures; Contesting neoliberalism: urban frontiers, Minnesota, MN: Guilford Press.

MAYUNGA, J. (2007). Understanding and applying the concept of community disaster resilience: a capitalbased approach. Summer Academy for Social Vulnerability and Resilience Building, 1, 16.

MINVU. (2008). Manual de Procedimientos Programa de Recuperación de Barrios. Gobierno de Chile.

MORROW, B. H. (2008). Community resilience: A social justice perspective.

http://doi.org/10.13140/RG.2.1.1278.9604 (Consulta: 23/02/2019).

ONEMI. (2016). Plan Estratégico Nacional para la Gestión del Riesgo de Desastres. Plataforma Nacional para la Reducción del Riesgo de Desastres.

PATEL, R. B., \& GLEASON, K. M. (2018). The association between social cohesion and community resilience in two urban slums of Port au Prince, Haiti. International Journal of Disaster Risk Reduction, 27, 161-167. https://doi.org/10.1016/j.ijdrr.2017.10.003.

ROBERTS, P. (2000). The evolution, definition and purpose of urban regeneration. In P. Roberts, P. W. Roberts, \& H. Sykes (Eds.), Urban Regeneration (p. 320). SAGE.

SMITH, M. K. (2011). Neighbourhoods and regeneration. Theory, practice, issues. In the encyclopaedia of informal education. Retrieved from www.infed.org/community/neighbourhoods and regeneration.htm(Consulta: 20/02/2019).

TOWNSHEND, I., AWOSOGA, O., KULIG, J., \& FAN, H. (2015). Social cohesion and resilience across communities that have experienced a disaster. Natural Hazards, 76(2), 913-938. https://doi.org/10.1007/s11069-014-1526-4 